Kragujevac Journal of Mathematics

Volume 44(1) (2020), Pages 7-26.

\title{
SOLUTION AND STABILITY OF A CUBIC TYPE FUNCTIONAL EQUATION: USING DIRECT AND FIXED POINT METHODS
}

\author{
V. GOVINDAN ${ }^{1}$, S. MURTHY ${ }^{2}$, AND M. SARAVANAN $^{3}$
}

\begin{abstract}
In this concept, we investigate the generalized Ulam-Hyers-Rassias stability for the new type of cubic functional equation of the form

$$
g\left(a x_{1}+b x_{2}+2 c x_{3}\right)+g\left(a x_{1}+b x_{2}-2 c x_{3}\right)+8 a^{3} g\left(x_{1}\right)+8 b^{3} g\left(x_{2}\right)
$$$$
=2 g\left(a x_{1}+b x_{2}\right)+4\left(g\left(a x_{1}+c x_{3}\right)+g\left(a x_{1}-c x_{3}\right)+g\left(b x_{2}+c x_{3}\right)+g\left(b x_{2}-c x_{3}\right)\right)
$$

by using direct and fixed point alternative.

\section{INTRODUCTION}

Sometime in modeling applied problems there may be a degree of uncertainty in the parameters used in the model or some measurements may be imprecise. Due to such features, we are tempted to consider the study of the functional equation in the alternative settings. One of the most interesting questions in the theory of functional equations, concerning the famous Ulam [38] stability problem, is as follows: when is it true that a mapping satisfying a functional equation approximately must be close to exact solution of the given functional equation?

In 1940, S. M. Ulam [39] raised the following question. Under what conditions does there exist an additive mapping near an approximately additive linear mappings? The case of approximately additive function was solved by D. H. Hyers [15] under certain assumptions. In 1978, a generalized version of the Theorem of Hyers for approximately linear mappings was given by Th. M. Rassias [34]. A number of mathematicians were attracted by the result of Th. M. Rassias. The stability concept that was introduced and investigated by Rassias is called the Hyers-Ulam-Rassias stability. One of the

Key words and phrases. Cubic functional equation, generalized Hyers-Ulam stability, fixed point. 2010 Mathematics Subject Classification. Primary: 39B52. Secondary: 32B72, 32B82.

DOI $10.46793 / \mathrm{KgJMat} 2001.007 \mathrm{G}$

Received: November 15, 2016.

Accepted: January 30, 2018. 
most famous functional equation is the additive functional equation

$$
f(x+y)=f(x)+f(y) .
$$

In 1821, it was first solved by A. L. Cauchy in the class of the continuous real valued functions. It is often called an additive Cauchy functional equation in honor of A. L. Cauchy [39]. The theory of additive functional equations in frequently applied to the development of the theories of the other functional equations. Consider the functional equation

$$
f(x+y)+f(x-y)=2 f(x)+2 f(y) .
$$

The quadratic function $f(x)=c x^{2}$ is a solution of this functional equation, so one can usually say that the above functional equation is quadratic $[3-6,20,21,27,30]$.

Recently, Bae, Lee and Park [32] established some stability results for the functional equation

$$
k f(x+k y)+f(k x-y)=\frac{k\left(k^{2}-1\right)}{2}(f(x+y)+f(x-y))+(k \pm 1) f(y),
$$

where $k \geq 2$ is a fixed integer, in the setting of non-Archimedean $L$-fuzzy normed spaces.

The Hyers-Ulam stability problem of the quadratic functional equation was first proved by F. Skof [37] for functions between a normed space and a Banach space. After wards, the result was extended by P. W. Cholewa [11] and S. Czerwisk [12].

The cubic function $g(x)=c x^{3}$ satisfies the functional equation

$$
g(2 x+y)+g(2 x-y)=2 g(x+y)+2 g(x-y)+12 g(x) .
$$

Hence, throughout this concept, we promise that equation (1.1) is called a cubic functional equation and every solution of equation (1.1) is said to be a cubic function. The stability result of equation (1.1) was obtained by K. W. Jun and H. M. Kim [17].

In this concept, we present the general solution and generalized Ulam-Hyers-Rassias stability of the new type of cubic functional equation of the form

$$
\begin{aligned}
& g\left(a x_{1}+b x_{2}+2 c x_{3}\right)+g\left(a x_{1}+b x_{2}-2 c x_{3}\right)+8 a^{3} g\left(x_{1}\right)+8 b^{3} g\left(x_{2}\right) \\
= & 2 g\left(a x_{1}+b x_{2}\right)+4\left(g\left(a x_{1}+c x_{3}\right)+g\left(a x_{1}-c x_{3}\right)+g\left(b x_{2}+c x_{3}\right)+\right. \\
& \left.g\left(b x_{2}-c x_{3}\right)\right) .
\end{aligned}
$$

The main goal of this concept is to obtain the generalized Hyers-Ulam-Rassias stability result for the functional equation (1.2) by using the direct and fixed point alternative $[7,13,18,19,22-26,29,33,35,36]$ in $[1,2,8-10,14,16,28,31]$.

For completeness, we will first investigate solution of the functional equation (1.2).

Proposition 1.1. Let $X$ and $Y$ be real vector spaces. A function $g: X \rightarrow Y$ satisfies the functional equation (1.1) if and only if $g: X \rightarrow Y$ also satisfies the functional equation (1.2). 
Proof. Substituting $(x, y)$ by $(0,0)$ in $(1.1)$ yields $g(0)=0$. Replacing $(x, y)$ by $(0, x)$ in (1.1), gives $g(-x)=-g(x)$ for all $x \in X$, which implies that $g$ is odd. Now, replacing $(x, y)$ by $(x, 0)$ in $(1.1)$, we obtain $g(2 x)=8 g(x)$, and replacing $(x, y)$ by $(x, x)$ in (1.1), we get that $g(3 x)=27 g(x)$ for all $x \in X$. Substituting $(x, y)$ by $(a x, a x+b y)$ in $(1.1)$, we have

$$
\begin{aligned}
g(2 a x+a x+b y)+g(2 a x-(a x+b y))= & 2 g(a x+a x+b y) \\
& +2 g(a x-(a x+b y))+12 g(a x), \\
g(3 a x+b y)+g(a x-b y)= & 2 g(2 a x+b y)+2 g(-b y)+12 g(a x), \\
g(3 a x+b y)+g(a x-b y)= & 2 g(2 a x+b y)-2 g(b y)+12 g(a x),
\end{aligned}
$$

for all $x, y \in X$. Replacing $(x, y)$ by $(a x, a x-b y)$ in $(1.1)$, we get

$$
\begin{aligned}
g(2 a x+a x-b y)+g(2 a x-(a x-b y))= & 2 g(a x+a x-b y) \\
& +2 g(a x-(a x-b y))+12 g(a x), \\
g(3 a x-b y)+g(a x+b y)= & 2 g(2 a x-b y)+2 g(b y)+12 g(a x),
\end{aligned}
$$

for all $x, y \in X$. Adding (1.3) and (1.4) and then using (1.1), we see that

$$
\begin{aligned}
& g(3 a x+b y)+g(a x-b y)+g(3 a x-b y)+g(a x+b y) \\
= & 2 g(2 a x+b y)-2 g(b y)+12 g(a x)+2 g(2 a x-b y)+2 g(b y)+12 g(a x), \\
& g(3 a x+b y)+g(3 a x-b y)+g(a x+b y)+g(a x-b y) \\
= & 2 g(2 a x+b y)+2 g(2 a x-b y)+24 g(a x), \\
& g(3 a x+b y)+g(3 a x-b y)+g(a x+b y)+g(a x-b y) \\
= & 2(2 g(a x+b y)+2 g(a x-b y)+12 g(a x))+24 g(a x), \\
& g(3 a x+b y)+g(3 a x-b y)+g(a x+b y)+g(a x-b y) \\
= & 4 g(a x+b y)+4 g(a x-b y)+24 g(a x)+24 g(a x), \\
& g(3 a x+b y)+g(3 a x-b y)+g(a x+b y)+g(a x-b y) \\
= & 4 g(a x+b y)+4 g(a x-b y)+48 g(a x), \\
& g(3 a x+b y)+g(3 a x-b y)=3 g(a x+b y)+3 g(a x-b y)+48 g(a x),
\end{aligned}
$$

for all $x, y \in X$. Now, replacing $(a x, b y)$ by $(a x+b y, a x-b y)$ in $(1.5)$, respectively, we have

$$
\begin{aligned}
& g(3(a x+b y)+(a x-b y))+g(3(a x+b y)-(a x-b y)) \\
= & 3 g((a x+b y)+(a x-b y))+3 g((a x+b y)-(a x-b y))+48 g(a x+b y), \\
& g(3 a x+3 b y+a x-b y)+g(3 a x+3 b y-a x+b y) \\
= & 3 g(2 a x)+3 g(2 b y)+48 g(a x+b y), \\
& g(4 a x+2 b y)+g(2 a x+4 b y)=3 g(2 a x)+3 g(2 b y)+48 g(a x+b y),
\end{aligned}
$$

for all $x, y \in X$, which, in view of the identity $g(2 x)=8 g(x)$, reduces to

$$
g(4 a x+2 b y)+g(2 a x+4 b y)
$$




$$
\begin{aligned}
= & 3(8 g(a x))+3(8 g(b y))+48 g(a x+b y), \\
& 8 g(2 a x+b y)+8 g(a x+2 b y)=24 g(a x)+24 g(b y)+48 g(a x+b y),
\end{aligned}
$$

and dividing by 8 , we get

$$
g(2 a x+b y)+g(a x+2 b y)=3 g(a x)+3 g(b y)+6 g(a x+b y),
$$

for all $x, y \in X$. Now, replacing $(a x, b y)$ by $(a x+3 b y, a x-3 b y)$ in $(1.6)$, we arrive to

$$
\begin{aligned}
& g(2(a x+3 b y)+(a x-3 b y))+g(a x+3 b y+2(a x-3 b y)) \\
&= 3 g(a x+3 b y)+3 g(a x-3 b y)+6 g(a x+3 b y+a x-3 b y), \\
& g(2 a x+6 b y+a x-3 b y)+g(a x+3 b y+2 a x-6 b y) \\
&= 3 g(a x+3 b y)+3 g(a x-3 b y)+6 g(2 a x), \\
& g(3 a x+3 b y)+g(3 a x-3 b y)=3 g(a x+3 b y)+3 g(a x-3 b y)+(6 \times 8) g(a x), \\
& 27 g(a x+b y)+27 g(a x-b y)=3 g(a x+3 b y)+3 g(a x-3 b y)+48 g(a x), \\
&(1.7) \quad 9 g(a x+b y)+9 g(a x-b y)=g(a x+3 b y)+g(a x-3 b y)+16 g(a x),
\end{aligned}
$$

for all $x, y \in X$. Let us interchange $a x$ in $b y$ and $b y$ in $a x$ in (1.7) to get the identities

$$
\begin{aligned}
& 9 g(a x+b y)+9 g(b y-a x)=g(3 a x+b y)+g(b y-3 a x)+16 g(b y), \\
& 9 g(a x+b y)-9 g(a x-b y)=g(3 a x+b y)-g(3 a x-b y)+16 g(b y),
\end{aligned}
$$

for all $x, y \in X$. Then, by adding (1.7) and (1.8), we get

$$
\begin{aligned}
& 9 g(a x+b y)+9 g(a x-b y)+9 g(a x+b y)-9 g(a x-b y) v \\
= & g(a x+3 b y) x+g(a x-3 b y)+16 g(a x) \\
& +g(3 a x+b y)-g(3 a x-b y)+16 g(b y), \\
18 g(a x+b y)= & g(a x+3 b y)+g(a x-3 b y)+g(3 a x+b y) \\
& -g(3 a x-b y)+16 g(a x)+16 g(b y),
\end{aligned}
$$

for all $x, y \in X$. Now, we interchange $a x$ with by and by with $a x$ in (1.5), respectively we get

$$
\begin{aligned}
& g(3 b y+a x)+g(3 b y-a x)=3 g(b y+a x)+3 g(b y-a x)+48 g(b y), \\
& g(a x+3 b y)-g(a x-3 b y)=3 g(a x+b y)-3 g(a x-b y)+48 g(b y),
\end{aligned}
$$

for all $x, y \in X$. Hence, according to (1.5) and (1.11), we obtain

$$
\begin{aligned}
& g(3 a x+b y)+g(3 a x-b y)=3 g(a x+b y)+3 g(a x-b y)+48 g(a x), \\
& g(a x+3 b y)-g(a x-3 b y)=3 g(a x+b y)-3 g(a x-b y)+48 g(b y) .
\end{aligned}
$$

Adding the above equations we get

$$
\begin{aligned}
& g(3 a x+b y)+g(3 a x-b y)+g(a x+3 b y)-g(a x-3 b y) \\
= & 6 g(a x+b y)+48 g(a x)+48 g(b y), \\
6 g(a x+b y)= & g(3 a x+b y)+g(3 a x-b y)+g(a x+3 b y)-g(a x-3 b y)
\end{aligned}
$$




$$
-48 g(a x)-48 g(b y)
$$

for all $x, y \in X$. Again by adding (1.10) and (1.12), we get

$$
\begin{aligned}
18 g(a x+b y)= & g(a x+3 b y)+g(a x-3 b y)+g(3 a x+b y) \\
& -g(3 a x-b y)-16 g(a x)-16 g(b y), \\
6 g(a x+b y)= & g(3 a x+b y)+g(3 a x-b y)+g(a x+3 b y) \\
& -g(a x-3 b y)-48 g(a x)-48 g(b y), \\
24 g(a x+b y)= & 2 g(a x+3 b y)+2 g(3 a x+b y) \\
& -32 g(a x)-32 g(b y), \\
12 g(a x+b y)= & g(a x+3 b y)+g(3 a x+b y)-16 g(a x)-16 g(b y), \\
(1.13) g(a x+3 b y)+g(3 a x+b y)= & 12 g(a x+b y)+16 g(a x)+16 g(b y),
\end{aligned}
$$

for all $x, y \in X$. Taking (1.5), we have

$$
\begin{aligned}
& g(3 a x+b y)+g(3 a x-b y)=3 g(a x+b y)+3 g(a x-b y)+48 g(a x), \\
& g(3 a x+c z)+g(3 a x-c z)=3 g(a x+c z)+3 g(a x-c z)+48 g(a x), \\
& g(3 b y+c z)+g(3 b y-c z)=3 g(b y+c z)+3 g(b y-c z)+48 g(b y), \\
& g(3 a x+c z)+g(3 a x-c z)+g(3 b y+c z)+g(3 b y-c z) \\
= & 3 g(a x+c z)+3 g(a x-c z)+48 g(a x) \\
& +3 g(b y+c z)+3 g(b y-c z)+48 g(b y), \\
& 16 g(3 a x+c z)+16 g(3 a x-c z)+16 g(3 b y+c z)+16 g(3 b y-c z) \\
= & 48 g(a x+c z)+48 g(a x-c z)+48 g(b y+c z) \\
& +48 g(b y-c z)+768 g(a x)+768 g(b y),
\end{aligned}
$$

for all $x, y \in X$. Also, replacing $(a x, b y)$ by $(3 a x+c z, 3 b y+c z)$ in (1.13), respectively we get

$$
\begin{aligned}
& g(a x+3 b y)+g(3 a x+b y)=12 g(a x+b y)+16 g(a x)+16 g(b y), \\
& g(3 a x+c z+3(3 b y+c z))+g(3(3 a x+c z)+3 b y+c z) \\
= & 12 g(3 a x+c z+3 b y+c z)+16 g(3 a x+c z)+g(3 b y+c z), \\
& g(3 a x+c z+9 b y+3 c z)+g(9 a x+3 c z+3 b y+c z) \\
= & 12 g(3 a x+c z+3 b y+c z)+16 g(3 a x+c z)+16 g(3 b y+c z), \\
& g(3 a x+4 c z+9 b y)+g(9 a x+4 c z+3 b y) \\
= & 12 g(3 a x+2 c z+3 b y)+16 g(3 a x+c z)+16 g(3 b y+c z),
\end{aligned}
$$

for all $x, y \in X$. Replacing $(a x, b y)$ by $(3 a x-c z, 3 b y-c z)$ in (1.13) we obtain

$$
\begin{aligned}
& g(a x+3 b y)+g(3 a x+b y)=12 g(a x+b y)+16 g(a x)+16 g(b y), \\
& g(3 a x-c z+3(3 b y-c z))+g(3(3 a x-c z)+3 b y-c z) \\
= & 12 g(3 a x-c z+3 b y-c z)+16 g(3 a x-c z)+g(3 b y-c z),
\end{aligned}
$$




$$
\begin{aligned}
& g(3 a x-c z+9 b y-3 c z)+g(9 a x-3 c z+3 b y-c z) \\
= & 12 g(3 a x-c z+3 b y-c z)+16 g(3 a x-c z)+16 g(3 b y-c z), \\
& g(3 a x-4 c z+9 b y)+g(9 a x-4 c z+3 b y) \\
= & 12 g(3 a x-2 c z+3 b y)+16 g(3 a x-c z)+16 g(3 b y-c z),
\end{aligned}
$$

for all $x, y \in X$. Using (1.15) and (1.16), we get the following identities

$$
\begin{aligned}
& g(3 a x+4 c z+9 b y)+g(9 a x+4 c z+3 b y)+g(3 a x-4 c z+9 b y) \\
& +g(9 a x-4 c z+3 b y) \\
= & 12 g(3 a x+2 c z+3 b y)+16 g(3 a x+c z)+16 g(3 b y+c z) \\
& +12 g(3 a x-2 c z+3 b y)+16 g(3 a x-c z)+16 g(3 b y-c z), \\
& g(3 a x+4 c z+9 b y)+g(9 a x+4 c z+3 b y)+g(3 a x-4 c z+9 b y) \\
& +g(9 a x-4 c z+3 b y)-12 g(3 a x+2 c z+3 b y)-12 g(3 a x-2 c z+3 b y)
\end{aligned}
$$$$
\text { for all } x, y \in X \text {. Using (1.5) we obtain }
$$$$
g(3 a x+b y)+g(3 a x-b y)=3 g(a x+b y)+3 g(a x-b y)+48 g(a x),
$$$$
g(3(a x+3 b y)+4 c z)+g(3(a x+3 b y)-4 c z)
$$$$
=3 g(a x+3 b y+4 c z)+3 g(a x+3 b y-4 c z)+48 g(a x+3 b y),
$$$$
g(3 a x+9 b y+4 c z)+g(3 a x+9 b y-4 c z)
$$

$$
=3 g(a x+3 b y+4 c z)+3 g(a x+3 b y-4 c z)+48 g(a x+3 b y),
$$

for all $x, y \in X$. Again using (1.5) it follows that

$$
\begin{aligned}
& g(3 a x+b y)+g(3 a x-b y), \\
= & 3 g(a x+b y)+3 g(a x-b y)+48 g(a x), \\
& g(3(3 a x+b y)+4 c z)+g(3(3 a x+b y)-4 c z) \\
= & 3 g(3 a x+b y+4 c z)+3 g(3 a x+b y-4 c z)+48 g(3 a x+b y), \\
& g(9 a x+3 b y+4 c z)+g(9 a x+3 b y-4 c z) \\
= & 3 g(3 a x+b y+4 c z)+3 g(3 a x+b y-4 c z)+48 g(3 a x+b y),
\end{aligned}
$$

for all $x, y \in X$. Adding (1.18) and (1.19), we obtain

$$
\begin{aligned}
& g(3 a x+9 b y+4 c z)+g(3 a x+9 b y-4 c z)+g(9 a x+3 b y+4 c z) \\
& +g(9 a x+3 b y-4 c z)=3 g(a x+3 b y+4 c z)+3 g(a x+3 b y-4 c z) \\
& +48 g(a x+3 b y)+3 g(3 a x+b y+4 c z) \\
& +3 g(3 a x+b y-4 c z)+48 g(3 a x+b y),
\end{aligned}
$$

for all $x, y \in X$. Then applying (1.20) in (1.17), we get

$$
\begin{aligned}
& 16 g(3 a x+c z)+16 g(3 b y+c z)+16 g(3 a x-c z)+16 g(3 b y-c z) \\
= & 3 g(a x+3 b y+4 c z)+3 g(a x+3 b y-4 c z)+48 g(a x+3 b y)
\end{aligned}
$$




$$
\begin{aligned}
& +3 g(3 a x+b y+4 c z)+3 g(3 a x+b y-4 c z) \\
& -12 g(3 a x+3 b y+2 c z)-12 g(3 a x+3 b y-2 c z)+48 g(3 a x+b y),
\end{aligned}
$$

for all $x, y \in X$. From (1.5), we obtain

$$
\begin{aligned}
& g(3 a x+b y)+g(3 a x-b y)=3 g(a x+b y)+3 g(a x-b y)+48 g(a x), \\
& g(3(a x+b y)+2 c z)+g(3(a x+b y)-2 c z)=3 g(a x+b y+2 c z) \\
& +3 g(a x+b y-2 c z)+48 g(a x+b y), \\
& g(3 a x+3 b y+2 c z)+g(3 a x+3 b y-2 c z) \\
= & 3 g(a x+b y+2 c z)+3 g(a x+b y-2 c z)+48 g(a x+b y),
\end{aligned}
$$

for all $x, y, z \in X$. Using (1.22) in (1.21), we get

$$
\begin{aligned}
& 16 g(3 a x+c z)+16 g(3 b y+c z)+16 g(3 a x-c z)+16 g(3 b y+c z) \\
= & 3 g(a x+3 b y+4 c z)+3 g(a x+3 b y-4 c z)+48 g(a x+3 b y) \\
& +3 g(3 a x+b y+4 c z)+3 g(3 a x+b y-4 c z)+48 g(3 a x+b y) \\
& -36 g(a x+b y+2 c z)-36 g(a x+b y-2 c z)-576 g(a x+b y), \\
& 16 g(3 a x+c z)+16 g(3 b y+c z)+16 g(3 a x-c z)+16 g(3 b y+c z) \\
= & 3 g(a x+3 b y+4 c z)+3 g(a x+3 b y-4 c z)+48 g(a x+3 b y) \\
& +3 g(3 a x+b y+4 c z)+3 g(3 a x+b y-4 c z) \\
& +48 g(3 a x+b y)-36 g(a x+b y+2 c z)-36 g(a x+b y-2 c z)-576 g(a x+b y),
\end{aligned}
$$

for all $x, y, z \in X$, which, by modifying of (1.14), yields to the relation

$$
\begin{aligned}
& 3 g(a x+3 b y+4 c z)+3 g(a x+3 b y-4 c z)+48 g(a x+3 b y) \\
& +3 g(3 a x+b y+4 c z)+3 g(3 a x+b y-4 c z)+48 g(3 a x+b y) \\
& -36 g(a x+b y+2 c z)-36 g(a x+b y-2 c z)-576 g(a x+b y) \\
= & 48 g(a x+c z)+48 g(a x-c z)+768 g(a x)+48 g(b y+c z) \\
& +48 g(b y-c z)+768 g(b y) .
\end{aligned}
$$

Then we obtain

$$
\begin{aligned}
& 3 g(a x+3 b y+4 c z)+3 g(a x+3 b y-4 c z)+48 g(a x+3 b y) \\
& +3 g(3 a x+b y+4 c z)+3 g(3 a x+b y-4 c z)+48 g(3 a x+b y) \\
= & 36 g(a x+b y+2 c z)+36 g(a x+b y-2 c z)+576 g(a x+b y)+48 g(a x+c z) \\
& +48 g(a x-c z)+768 g(a x)+48 g(b y+c z)+48 g(b y-c z)+768 g(b y),
\end{aligned}
$$

for all $x, y, z \in X$. With the concept of (1.13) and (1.5), the left side of (1.14) can be written in the form

$$
\begin{aligned}
& g(3 a x+b y)+g(3 a x-b y)=3 g(a x+b y)+3 g(a x-b y)+48 g(a x), \\
& g(3(3 a x+b y)+2 c z)+g(3(3 a x+b y)-2 c z) \\
= & 3 g(3 a x+b y+2 c z)+3 g(3 a x+b y-2 c z)+48 g(3 a x+b y),
\end{aligned}
$$




$$
\begin{aligned}
& g(9 a x+3 b y+2 c z)+g(9 a x+3 b y-2 c z)=3 g(3 a x+b y+2 c z) \\
& +3 g(3 a x+b y-2 c z)+48 g(3 a x+b y), \\
& g(3(a x+3 b y)+2 c z)+g(3(a x+3 b y)-2 c z) \\
= & 3 g(a x+3 b y+2 c z)+3 g(a x+3 b y-2 c z)+48 g(a x+3 b y), \\
& g(3 a x+9 b y+2 c z)+g(3 a x+9 b y-2 c z)=3 g(a x+3 b y+2 c z) \\
& +3 g(a x+3 b y-2 c z)+48 g(a x+3 b y) .
\end{aligned}
$$

Adding (1.24) and (1.25), we get

$$
\begin{aligned}
& g(3 a x+9 b y+2 c z)+g(3 a x+9 b y-2 c z)+g(9 a x+3 b y+2 c z) \\
& +g(9 a x+3 b y-2 c z)=3 g(a x+3 b y+2 c z)+3 g(a x+3 b y-2 c z) \\
& +48 g(a x+3 b y)+3 g(3 a x+b y+2 c z) \\
& +3 g(3 a x+b y-2 c z)+48 g(3 a x+b y), \\
& g(3 a x+9 b y+2 c z)+g(3 a x+9 b y-2 c z)+g(9 a x+3 b y+2 c z) \\
& +g(9 a x+3 b y-2 c z)-48 g(3 a x+b y)-48 g(a x+3 b y) \\
= & 3 g(3 a x+b y+2 c z)+3 g(a x+3 b y-2 c z)+3 g(a x+3 b y+2 c z) \\
& +3 g(a x+3 b y-2 c z), \\
& g(3 a x+9 b y+2 c z)+g(3 a x+9 b y-2 c z)+g(9 a x+3 b y+2 c z) \\
& +g(9 a x+3 b y-2 c z)-12 g(3 a x+3 b y)-12 g(3 a x+3 b y) \\
= & 3 g(a x+3 b y+2 c z)+3 g(a x+3 b y-2 c z) \\
& +3 g(a x+3 b y+2 c z)+3 g(a x+3 b y-2 c z),
\end{aligned}
$$

for all $x, y, z \in X$. Using (1.26), we get the identity

$$
\begin{aligned}
& 16 g(3 a x+c z)+16 g(3 b y+c z)+16 g(3 a x-c z)+16 g(3 b y-c z) \\
= & 3 g(a x+3 b y+2 c z)+3 g(a x+3 b y-2 c z)+48 g(a x+3 b y) \\
& +3 g(3 a x+b y+2 c z)-648 g(a x+b y)+48 g(3 a x+b y),
\end{aligned}
$$

for all $x, y, z \in X$. Replacing $z$ by $2 z$ in (1.27) and then using (1.23), we get

$$
\begin{aligned}
& 16 g(3 a x+2 c z)+16 g(3 b y+2 c z)+16 g(3 a x-2 c z)+16 g(3 b y-2 c z) \\
= & 3 g(a x+3 b y+4 c z)+3 g(a x+3 b y-4 c z)+48 g(a x+3 b y) \\
& +3 g(3 a x+b y+4 c z)+3 g(3 a x+b y-4 c z) \\
& -648 g(a x+b y)+48 g(3 a x+b y),
\end{aligned}
$$

for all $x, y \in X$. Using (1.28) in (1.23), we obtain

$$
\begin{aligned}
& 16 g(3 a x+2 c z)+16 g(3 b y+2 c z)+16 g(3 a x-2 c z)+16 g(3 b y-2 c z) \\
= & 48 g(a x+c z)+48 g(a x-c z)+768 g(a x)+48 g(b y+c z) \\
& +48 g(b y-c z)+768 g(b y)+36 g(a x+b y+2 c z) \\
& +36 g(a x+b y-2 c z)+576 g(a x+b y)-648 g(a x+b y),
\end{aligned}
$$


for all $x, y, z \in X$. Again making use of (1.13) and (1.5), we get

$$
\begin{aligned}
& 16 g(3 a x+2 c z)+16 g(3 b y+2 c z)+16 g(3 a x-2 c z)+16 g(3 b y-2 c z) \\
= & g(12 a x+4 c z)+g(12 a x-4 c z)-12 g(6 a x)+g(12 b y+4 c z) \\
& +g(12 b y-4 c z)-12 g(6 b y) \\
= & 64 g(3 a x+c z)+64 g(3 a x-c z)-2592 g(a x)+64 g(3 b y+c z) \\
& +64 g(3 b y-c z)-2592 g(b y) \\
= & 64(g(3 a x+c z)+g(3 a x-c z)+g(3 b y+c z)+g(3 b y-c z)) \\
& -2592 g(a x)-2592 g(b y) \\
= & 64(3 g(a x+c z)+3 g(a x-c z)+48 g(a x)+3 g(b y+c z)+3 g(b y-c z) \\
& +48 g(b y))-2592 g(a x)-2592 g(b y),
\end{aligned}
$$

for all $x, y, z \in X$. Using (1.30) we have the following reduction

$$
\begin{aligned}
& 16 g(3 a x+2 c z)+16 g(3 b y+2 c z)+16 g(3 a x-2 c z)+16 g(3 b y-2 c z) \\
= & 192 g(a x+c z) 192 g(a x-c z)-480 g(a x) \\
& +192 g(b y+c z)+192 g(b y-c z)-480 g(b y),
\end{aligned}
$$

for all $x, y, z \in X$. Finally, if we compare (1.31) with (1.29), we can conclude that

$$
\begin{aligned}
& 48 g(a x+c z)+48 g(b y+c z)+48 g(a x-c z)+48 g(b y-c z)+768 g(a x)+768 g(b y) \\
& +36 g(a x+b y+2 c z)+36 g(a x+b y-2 c z)+576 g(a x+b y)-648 g(a x+b y) \\
= & 192 g(a x+c z)+192 g(a x-c z)-480 g(a x)+192 g(b y+c z)+192 g(b y-c z) \\
& -480 g(b y), \\
& 36 g(a x+b y+2 c z)+36 g(a x+b y-2 c z)=192 g(a x+c z)-48 g(a x+c z) \\
& +192 g(a x-c z)-48 g(a x-c z)+480 g(a x)-768 g(a x)-192 g(b y+c z) \\
& -48 g(b y+c z)+192 g(b y-c z)-48 g(b y-c z)+480 g(b y)-768 g(b y) \\
& +72 g(a x+b y), \\
& 36 g(a x+b y+2 c z)+36 g(a x+b y-2 c z)=144 g(a x+c z)+144 g(a x-c z) \\
& +144 g(b y+c z)+144 g(b y-c z)+72 g(a x+b y)-288 g(a x)-288 g(b y), \\
& g(a x+b y+2 c z)+g(a x+b y-2 c z)=2 g(a x+b y)+4 g(a x+c z) \\
& +4 g(a x-c z)+4 g(b y+c z)+4 g(b y-c z)-8 g(a x)-8 g(b y),
\end{aligned}
$$

for all $x, y, z \in X$. By considering $g(a x)=a^{3} g(x)$, we get

$$
\begin{aligned}
& g(a x+b y+2 c z)+g(a x+b y-2 c z)=2 g(a x+b y) \\
& +4(g(a x+c z)+g(a x-c z)+g(b y+c z)+g(b y-c z))-8 a^{3} g(x)-8 b^{3} g(y),
\end{aligned}
$$

for all $x, y, z \in X$, which implies that $g$ is cubic. Conversely, suppose that $g: X \rightarrow Y$ satisfies the functional equation (1.1). Putting $x=y=z=0$ in (1.2) we get $g(0)=0$. Changing $(x, y, z)$ by $\left(\frac{-x}{a}, \frac{x}{b}, \frac{x c}{2}\right)$ in the result we get $g(-x)=-g(x)$, which implies 
that $g$ is odd. Replacing $y=0$ in (1.2) and employing the fact that $g$ is odd, we obtain that

$$
\begin{aligned}
& g(a x+2 c z)+g(a x-2 c z)=2 g(a x)+4 g(a x+c z)+g(a x-c z) \\
& +4 g(c z)+4 g(-c z)-8 a^{3} g(x), \\
& g(a x+c z)+g(a x-2 c z)=-6 a^{3} g(x)+4 g(a x+c z)+4 g(a x-c z), \\
& g(a x+2 c z)+g(a x-2 c z)=-6 a^{3} g(x)+4 g(a x+c z)+4 g(a x-c z),
\end{aligned}
$$

for all $x, y, z \in X$. Replacing $(x, y, z)$ by $(x, 0,0)$ in $(1.6)$, we get

$$
g(a x)=a^{3} g(x)
$$

since

$$
\begin{aligned}
g(a x)+g(a x) & =2 g(a x)+4 g(a x)+4 g(a x)-8 a^{3} g(x), \\
2 g(a x) & =10 g(a x)-8 a^{3} g(x), \\
2 g(a x)-10 g(a x) & =-8 a^{3} g(x), \\
-8 g(a x) & =-8 a^{3} g(x), \\
g(a x) & =a^{3} g(x) .
\end{aligned}
$$

So we replace $x$ by $2 x$ in (1.32) and we get

$$
\begin{aligned}
g(2 a x+2 c z)+g(2 a x-2 c z) & =-6 a^{3} g(2 x)+4 g(2 a x+c z)+4 g(2 a x-c z), \\
8 g(a x+c z)+8 g(a x-c z) & =-48 a^{3} g(x)+4 g(2 a x+c z)+4 g(2 a x-c z), \\
2 g(a x+c z)+2 g(a x-c z) & =-12 a^{3} g(x)+g(2 a x+c z)+g(2 a x-c z), \\
g(2 a x+c z)+g(2 a x-c z) & =12 a^{3} g(x)+2 g(a x+c z)+2 g(a x-c z),
\end{aligned}
$$

and

$$
g(2 x+y)+g(2 x-y)=12 g(x)+2 g(x+y)+2 g(x-y),
$$

for all $x, y, z \in X$, which implies that $g$ is cubic. This completes the proof.

In this section, we present the generalized Hyers-Ulam-Rassias stability of the function (1.6).

Theorem 1.1. Let $j \in\{-1,1\}$ and $\alpha: X^{3} \rightarrow[0, \infty)$ be a function such that

$$
\sum_{k=0}^{\infty} \frac{\alpha\left(a^{k j} x, a^{k j} y, a^{k j} z\right)}{a^{3 k j}}
$$

converges in $\mathbb{R}$ and

$$
\sum_{k=0}^{\infty} \frac{\alpha\left(a^{k j} x, a^{k j} y, a^{k j} z\right)}{a^{3 k j}}=0,
$$

for all $x, y \in X$. Let $g: X \rightarrow Y$ be an odd function satisfying the inequality

$$
\left\|D_{g}(x, y, z)\right\| \leq \alpha(x, y, z),
$$


for all $x, y, z \in X$. Then there exists a unique cubic mapping $C: X \rightarrow Y$ which satisfies the functional equation (1.6) and

$$
\|f(x)-C(x)\| \leq \frac{1}{8 a^{3}} \sum_{k=\frac{i-j}{2}}^{\infty} \frac{\alpha\left(a^{k j} x, 0,0\right)}{a^{3 k j}},
$$

for all $x \in X$. The mapping $C(x)$ is defined by

$$
C(x)=\lim _{n \rightarrow \infty} \frac{g\left(a^{k j} x\right)}{a^{3 k j}},
$$

for all $x \in X$.

Proof. Assume that $j=1$. Replacing $(x, y, z)$ by $(x, 0,0)$ in $(1.34)$, we get

$$
\left\|8 g(a x)-8 a^{3} g(x)\right\| \leq \alpha(x, 0,0),
$$

for all $x \in X$. From (1.36) it follows that

$$
\left\|\frac{g(a x)}{a^{3}}-g(x)\right\| \leq \frac{1}{8 a^{3}} \alpha(x, 0,0),
$$

for all $x \in X$. Replacing $x$ by $a x$ in (1.37) and dividing by $a^{3}$, we obtain

$$
\begin{aligned}
\left\|\frac{g(a(a x))}{a^{6}}-\frac{g(a x)}{a^{3}}\right\| & \leq \frac{1}{8 a^{6}} \alpha(a x, 0,0), \\
\left\|\frac{g\left(a^{2} x\right)}{a^{6}}-\frac{g(a x)}{a^{3}}\right\| & \leq \frac{1}{8 a^{6}} \alpha(a x, 0,0),
\end{aligned}
$$

for all $x \in X$. From the identity (1.37) and (1.38), it follows that

$$
\begin{aligned}
\left\|\frac{g\left(a^{2} x\right)}{a^{6}}-g(x)\right\| & \leq \frac{1}{8 a^{3}} \alpha(x, 0,0)+\frac{1}{8 a^{6}} \alpha(a x, 0,0) \\
& \leq \frac{1}{8 a^{3}}\left\{\alpha(x, 0,0)+\frac{1}{a^{3}} \alpha(a x, 0,0)\right\} \\
& \leq \sum_{k=0}^{n-1} \frac{1}{8 a^{3}}\left\{\frac{\alpha\left(a^{k} x, 0,0\right)}{a^{3 k}}\right\} \\
\left\|\frac{g\left(a^{n} x\right)}{a^{3 n}}-g(a x)\right\| & \leq \frac{1}{8 a^{3}} \sum_{k=0}^{n-1}\left\{\frac{\alpha\left(a^{k} x, 0,0\right)}{a^{3 k}}\right\},
\end{aligned}
$$

for all $x \in X$. We prove the convergence of the sequence $\left\{\frac{g\left(a^{k} x\right)}{a^{3 k}}\right\}$ for all $x \in X$. Replacing $x$ by $a^{m} x$ and dividing by $a^{m}$ in (1.39), we obtain

$$
\left\|\frac{g\left(a^{m} x\right)}{a^{3 m}}-\frac{g\left(a^{m+n} x\right)}{a^{3(m+n)}}\right\| \leq \frac{1}{8 a^{3}} \sum_{k=0}^{n-1}\left\{\frac{\alpha\left(a^{m+n} x, 0,0\right)}{a^{3(m+n)}}\right\},
$$


for all $x \in X$. Hence, the sequence $\left\{\frac{g\left(a^{m} x\right)}{a^{3 m}}\right\}$ is a Cauchy sequence. Since $Y$ is complete normed space, there exists a mapping $C: X \rightarrow Y$ such that

$$
C(x)=\lim _{n \rightarrow \infty} \frac{g\left(a^{k j} x\right)}{a^{3 k j}},
$$

for all $x \in X$. Letting $k \rightarrow \infty$ in (1.39), we see that (1.34) holds for $x \in X$. To prove that $C$ satisfies (1.6), we replace $(x, y, z)$ by $\left(a^{n} x, a^{n} y, a^{n} z\right)$ and divide (1.34) by $a^{3 n}$, which gives that

$$
\frac{1}{a^{3 n}}\left\|D_{g}\left(a^{n} x, a^{n} y, a^{n} z\right)\right\| \leq \frac{1}{a^{3 n}} \alpha\left(a^{n} x, a^{n} y, a^{n} z\right)
$$

for all $x, y, z \in X$. As $n$ approaches to $\infty$ in the above inequality and using the definition of $C(x)$, we have $D C(x, y, z)=0$. Hence, $C$ satisfies (1.6) for all $x, y, z \in X$.

We will show that $C$ is unique. Let $B(x)$ be another cubic mapping satisfying (1.6) and (1.35), such that

$$
\begin{aligned}
\|C(x)-B(x)\| & =\frac{1}{a^{3 n}}\left\|c\left(a^{n} x\right)-B\left(a^{n} x\right)\right\| \\
& \leq \frac{1}{a^{3 n}}\left\{\left\|C\left(a^{n} x\right)-g\left(a^{n} x\right)\right\|+\left\|g\left(a^{n} x\right)-B\left(a^{n} x\right)\right\|\right\} \\
& \leq \frac{1}{8 a^{3 n}} \sum_{k=0}^{\infty} \frac{\alpha\left(a^{m+n} x, 0,0,\right)}{a^{3(m+n)}} \rightarrow 0 \quad \text { as } n \rightarrow \infty
\end{aligned}
$$

for all $x \in X$. Hence, $C$ is unique. Now, replacing $x$ by $\frac{x}{a}$ in (1.34), we get

$$
\begin{aligned}
\left\|8 g(a x)-8 a^{3} g(x)\right\| & \leq \alpha\left(\frac{x}{a}, 0,0\right), \\
\left\|g(a x)-a^{3} g(x)\right\| & \leq \frac{1}{8} \alpha\left(\frac{x}{a}, 0,0\right),
\end{aligned}
$$

for all $x \in X$. The remaining part of the proof of this theorem for $j=1$ with replacing $x$ by $\frac{x}{a}$ in (1.37) is similar. Also, we can prove the theorem for $j=-1$ in the same manner. This completes the proof of the theorem.

Corollary 1.1. Let $\lambda$ and $q$ be a non-negative real numbers. Let an odd function $g: X \rightarrow Y$ satisfying the inequality

$$
\|D g(x, y, z)\| \leq\left\{\begin{array}{l}
\lambda, \\
\lambda\left\{\|x\|^{q}+\|y\|^{q}+\|z\|^{q}\right\}, \\
\lambda\left\{\|x\|^{q}\|y\|^{q}\|z\|^{q}+\|x\|^{3 q}+\|y\|^{3 q}+\|z\|^{3 q}\right\},
\end{array}\right.
$$


for all $x, y, z \in X$. Then there exists a unique cubic mapping $C: X \rightarrow Y$ such that

$$
\|g(x)-C(x)\| \leq\left\{\begin{array}{l}
\frac{1}{8} \cdot \frac{1}{a^{3}-1}, \\
\frac{\lambda}{8} \cdot \frac{\|x\|^{q}}{\left|a^{3}-a^{q}\right|} \\
\frac{\lambda}{8} \cdot \frac{\|x\|^{3 q}}{\left|a^{3}-a^{3 q}\right|}
\end{array}\right.
$$

for all $x \in X$.

Proof. Setting

$$
\alpha(x, y, z) \leq\left\{\begin{array}{l}
\lambda, \\
\lambda\left\{\|x\|^{q}+\|y\|^{q}+\|z\|^{q}\right\} \\
\lambda\left\{\|x\|^{q}\|y\|^{q}\|z\|^{q}+\|x\|^{3 q}+\|y\|^{3 q}+\|z\|^{3 q}\right\}
\end{array}\right.
$$

for all $x \in X$ and using $\alpha(x, y, z)$ in Theorem 1.1, we obtain desired result.

In this section, we investigate the generalized Ulam-Hyers-Rassias stability of the functional equation (1.6).

Theorem 1.2 (The Alternative of Fixed Point, [29]). Suppose that complete generalized metric space $(\tau, d)$ and a strictly contractive mapping $T: \tau \rightarrow \tau$ with Lipchitz constant $L$ are given. Then for each given $x \in \tau$, either

$$
d\left(T^{n} x, T^{n+1} x\right)=\infty
$$

for all $n \geq 0$ or there exists a natural number $n_{0}$ such that

(a) $d\left(T^{n} x, T^{n+1} x\right)<\infty$ for all $n \geq 0$;

(b) the sequence $\left\{T^{n} x\right\}$ is convergent to a fixed point $y^{*}$ of $T$;

(c) $y^{*}$ is the unique fixed point of $T$ in the set

$$
Y=\left\{y \in Y: d\left(P^{n_{0}} x, y\right)<\infty\right\}
$$

(d) $d\left(y^{*}, y\right) \leq \frac{1}{1-L} d(y, P y)$ for all $y \in Y$.

Utilizing the above mentioned fixed point alternative, we now obtain our main results, that is the generalized Hyers-Ulam-Rassias stability of the functional equation (1.6).

From now on, let $X$ be a real vector space and $Y$ be a real Banach space. For given mapping $g: X \rightarrow Y$, we get

$$
\begin{aligned}
D g(x, y, z)= & g(a x+b y+2 c z)+g(a x+b y-2 c z)-2 g(a x+b y)-4 g(a x+c z) \\
& -4 g(a x-c z)-4 g(b y+c z)-4 g(b y-c z)+8 a^{3} g(x)+8 b^{3} g(y),
\end{aligned}
$$

for all $x, y, z \in X$. Let $\psi: X \times X \times X \rightarrow[0, \infty)$ be a function such that

$$
\lim _{n \rightarrow \infty} \frac{\psi\left(\mu_{i}^{k} x, \mu_{i}^{k} y, \mu_{i}^{k} z\right)}{\mu_{i}^{3 k}}=0,
$$


for all $x, y, z \in X$, where

$$
\mu_{i}= \begin{cases}2, & i=0 \\ \frac{1}{2}, & i=1 .\end{cases}
$$

Theorem 1.3. Suppose that function $g: X \rightarrow Y$ satisfies the functional inequality

$$
\|D g(x, y, z)\| \leq \psi(x, y, z),
$$

for all $x, y, z \in X$. If there exists $L=L(i)$ such that function

$$
x \mapsto \beta(x)=\frac{1}{2} \alpha\left(\frac{x}{a}, 0,0\right)
$$

has the property

$$
\frac{1}{\mu_{i}^{3}} \beta\left(\mu_{i} x\right)=L \beta(x)
$$

for all $x \in X$, then there exists a unique cubic function $c: X \rightarrow Y$ that satisfies the functional equation (1.6) and

$$
\|g(x)-c(x)\| \leq \frac{L^{1-i}}{1-L} \beta(x)
$$

for all $x \in X$.

Proof. Consider the set $W=\{p / P: X \rightarrow \beta, p(0)=0\}$ and introduced generalized metric on $X$

$$
d(p, q)=\inf \{k \in(0, \infty):\|p(x)-q(x)\| \leq k \beta(x), x \in X\} .
$$

It is easy to see that $(X, d)$ is complete. Define $T: X \rightarrow X$ by

$$
T p(x)=\frac{1}{\mu_{i}^{3}} p\left(\mu_{i}\right)
$$

for all $x \in X$. Now, for $p, q \in X$, we have

$$
\begin{aligned}
d(p, q) & \leq k, \quad x \in W, \\
\mid\|p(x)-q(x)\| & \leq k \beta(x), \quad x \in W, \\
\left\|\frac{1}{\mu_{i}^{3}} p\left(\mu_{i} x\right)-\frac{1}{\mu_{i}^{3}} q\left(\mu_{i} x\right)\right\| & \leq \frac{1}{\mu_{i}^{3}} k \beta\left(\mu_{i} x\right), \\
\|T p(x)-T q(x)\| & \leq L k \beta(x), \quad x \in W, \\
d(T p, T q) & \leq L k, \quad x \in W .
\end{aligned}
$$

This implies that $d(T p, T q) \leq L d(p, q)$ for all $p, q \in X$. That is, $T$ is strictly contractive mapping on $X$ with Lipschitz constant $L$. From (1.36) it follows that

$$
\left\|8 g(a x)-8 a^{3} g(x)\right\| \leq \alpha(x, 0,0),
$$


for all $x \in X$. From (1.43) it follows that

$$
\left\|\frac{g(a x)}{a^{3}}-g(x)\right\| \leq \frac{1}{8 a^{3}} \alpha(x, 0,0),
$$

for all $x \in X$. Using (1.42), for the case $i=0$, this reduces to

$$
\left\|g(x)-\frac{g(a x)}{a^{3}}\right\| \leq \frac{1}{4} \beta(x),
$$

for all $x \in X$, that is

$$
d\left(g_{a}, T g_{a}\right) \leq \frac{1}{4}=L=L^{1}<\infty .
$$

Again replacing $x$ by $\frac{x}{a}$ in $(1.43)$, we get

$$
\left\|g(x)-a^{3} g\left(\frac{x}{a}\right)\right\| \leq \frac{1}{8} \alpha\left(\frac{x}{a}, 0,0\right),
$$

for all $x \in X$. Using (1.42) for the case $i=1$, this reduces to

$$
\left\|g(x)-a^{3} g\left(\frac{x}{a}\right)\right\| \leq \frac{1}{4} \beta(x),
$$

for all $x \in X$, that is $d\left(g_{a}, T g_{a}\right) \leq 1$. This implies that $d\left(g_{a}, T g_{a}\right) \leq 1=L^{0}<\infty$. In the above case, we write $d\left(g_{a}, T g_{a}\right) \leq L^{1-i}$. Therefore, the first two conditions (a) and (b) of the Alternative fixed point theorem holds for $T$, and it follows that there exists a fixed point $C$ of $T$ in $X$ such that

$$
C(x)=\lim _{k \rightarrow \infty} \frac{g\left(\mu_{i}^{k} x\right)}{\mu_{i}^{3 k}}, \quad \text { for all } x \in X .
$$

In order to prove that $C: X \rightarrow Y$ is cubic we replace $(x, y, z)$ by $\left(\mu_{i}^{k} x, \mu_{i}^{k} y, \mu_{i}^{k} z\right)$ and divide (1.41) by $\mu_{i}^{3 k}$. From that, using (1.40) and (1.44), we see that $C$ satisfies (1.6) for all $x, y, z \in X$. Hence, $C$ satisfies the functional equation (1.6).

By fixed point condition (2), $C$ is the unique fixed point of $T$ in the set

$$
Y=\left\{g_{a} \in X: d\left(T g_{a}, C\right)<\infty\right\} .
$$

Using the fixed point alternative result, $C$ is the unique function such that

$$
\left\|g_{a}(x)-C(x)\right\| \leq k \beta(x),
$$

for all $x \in X$ and $k>0$. Finally, by (4), we obtain

$$
d\left(g_{a}, C\right) \leq \frac{1}{1-L} d\left(g_{a}, T g_{a}\right)
$$

That is, we have

Hence, we conclude that

$$
d\left(g_{a}, C\right) \leq \frac{L^{1-i}}{1-L}
$$

$$
\|g(x)-C(x)\| \leq \frac{L^{1-i}}{1-L} \beta(x)
$$


for all $x \in X$. This completes the proof of the theorem.

Corollary 1.2. Let $g: X \rightarrow Y$ be a mapping and let $t \gamma$ and $p$ be real numbers such that

$$
\|D g(x, y, z)\| \leq\left\{\begin{array}{l}
\gamma, \\
\gamma\left\{\|x\|^{p}+\|y\|^{p}+\|z\|^{p}\right\}, \\
\gamma\left\{\|x\|^{p}\|y\|^{p}\|z\|^{p}+\left(\|x\|^{3 p}+\|y\|^{3 p}+\|z\|^{3 p}\right)\right\},
\end{array}\right.
$$

for all $x, y, z \in X$. Then there exist a unique cubic mapping $C: X \rightarrow Y$ such that

$$
\|g(x)-C(x)\| \leq \begin{cases}\frac{\gamma}{8} \cdot \frac{1}{a^{3}-1}, \\ \frac{\gamma}{8} \cdot \frac{\|x\|^{p}}{a^{3}-a^{p}}, & p \neq 3, \\ \frac{\gamma}{8} \cdot \frac{\|x\|^{3 q}}{a^{3}-a^{3 q}}, & p \neq 1,\end{cases}
$$

for all $x \in X$.

Proof. Set

$$
\alpha(x, y, z)=\left\{\begin{array}{l}
\gamma \\
\gamma\left\{\|x\|^{p}+\|y\|^{p}+\|z\|^{p}\right\}, \\
\gamma\left\{\|x\|^{p}\|y\|^{p}\|z\|^{p}+\left(\|x\|^{3 p}+\|y\|^{3 p}+\|z\|^{3 p}\right)\right\},
\end{array},\right.
$$

for all $x \in X$. Now,

$$
\begin{aligned}
& \frac{\alpha\left(\mu_{i}^{k} x, \mu_{i}^{k} y, \mu_{i}^{k} z\right)}{\mu_{i}^{3 k}} \\
&=\left\{\begin{array}{l}
\frac{\gamma}{\mu_{\dot{\gamma}}^{3 k}}, \\
\frac{\mu_{i}^{3 k}}{\gamma}\left\{\left\|\mu_{i}^{k} x\right\|^{p}+\left\|\mu_{i}^{k} y\right\|^{p}+\left\|\mu_{i}^{k} z\right\|^{p}\right\}, \\
\frac{\mu_{i}^{3 k}}{2}\left\{\mu_{i}^{k} x\left\|^{p}\right\| \mu_{i}^{k} y\left\|^{p}\right\| \mu_{i}^{k} z \|^{p}+\left(\left\|\mu_{i}^{k} x\right\|^{3 p}+\left\|\mu_{i}^{k} y\right\|^{3 p}+\left\|\mu_{i}^{k} z\right\|^{3 p}\right)\right\},
\end{array}\right. \\
& \rightarrow \begin{cases}0, & k \rightarrow \infty, \\
0, & k \rightarrow \infty, \\
0, & k \rightarrow \infty .\end{cases}
\end{aligned}
$$

That is, (1.40) holds. But we have $\beta(x)=\frac{1}{2} \alpha\left(\frac{x}{a}, 0,0\right)$. Hence,

$$
\beta(x)=\frac{1}{2} \alpha\left(\frac{x}{a}, 0,0\right)=\left\{\begin{array}{l}
\frac{\gamma}{8}, \\
\frac{\gamma}{2^{3} a^{p}}\|x\|^{p}, \\
\frac{\gamma}{2^{3} a^{3 p}}\|x\|^{3 p} .
\end{array}\right.
$$


Also,

$$
\frac{1}{\mu_{i}^{3}} \beta(\mu, x)=\left\{\begin{array}{l}
\frac{\gamma}{8 \mu_{i}^{3}}, \\
\frac{\gamma}{8 \mu_{i}^{3}}\left\|\mu_{i}\right\|^{p}, \\
\frac{\gamma}{8 \mu_{i}^{3}}\left\|\mu_{i}\right\|^{3 p},
\end{array}=\left\{\begin{array}{l}
\mu_{i}^{-3} \beta(x), \\
\mu_{i}^{p-3} \beta(x), \\
\mu_{i}^{3 p-3} \beta(x) .
\end{array}\right.\right.
$$

Hence, the inequality (1.42) holds.

Case (i). $L=a^{-3}, i=0$,

$$
\begin{aligned}
\|g(x)-C(x)\| & \leq \frac{L^{1-i}}{1-L} \beta(x) \leq \frac{\left(a^{-3}\right)^{1-0}}{1-a^{-3}} \frac{\gamma}{8} \\
& \leq \frac{a^{-3}}{1-\frac{1}{a^{3}}} \cdot \frac{\gamma}{8} \leq \frac{\gamma}{8} \cdot \frac{1}{a^{3}-1} .
\end{aligned}
$$

Case (ii). $L=\left(\frac{1}{a^{3}}\right)^{-1}, i=1$,

$$
\begin{aligned}
\|g(x)-C(x)\| & \leq \frac{L^{1-i}}{L} \beta(x) \leq \frac{\left(a^{3}\right)^{1-1}}{1-a^{3}} \frac{\gamma}{8} \\
& \leq \frac{1}{1-a^{3}} \cdot \frac{\gamma}{8} \leq \frac{\gamma}{8} \cdot \frac{1}{1-a^{3}} .
\end{aligned}
$$

Case (iii). $L=a^{p-3}, p<3, i=0$

$$
\begin{aligned}
\|g(x)-C(x)\| & \leq \frac{\left(a^{p-3}\right)^{1-0}}{1-a^{p-3}} \cdot \frac{\gamma}{2^{3} a^{p}}\|x\|^{p} \\
& \leq \frac{a^{p} a^{-3}}{1-\frac{a^{p}}{a^{3}}} \cdot \frac{\gamma}{2^{3} a^{p}}\|x\|^{p}, \\
\|g(x)-C(x)\| & \leq \frac{\|x\|^{p} \gamma}{8} \cdot \frac{1}{a^{3}-a^{p}} .
\end{aligned}
$$

Case (iv). $L=\left(\frac{1}{a}\right)^{p-3}, p>3, i=1, L=a^{3-p}, p>3, i=1$,

$$
\begin{aligned}
\|g(x)-C(x)\| & \leq \frac{L^{1-i}}{1-L} \beta(x) \\
& \leq \frac{\left(a^{3-p}\right)^{1-1}}{1-a^{3-p}} \cdot \frac{\gamma}{2^{3} a^{p}}\|x\|^{p}, \\
\|g(x)-C(x)\| & \leq \frac{a^{p}}{a^{p}-a^{3}} \cdot \frac{\gamma}{8 a^{p}}\|x\|^{p} \leq \frac{\|x\|^{p} \cdot \gamma}{8} \cdot \frac{1}{a^{p}-a^{3}} .
\end{aligned}
$$

Case (v). $L=a^{3 p-3}, p<1, i=0$

$$
\|g(x)-C(x)\| \leq \frac{L^{1-i}}{L} \beta(x) \leq \frac{\left(a^{3 p-3}\right)^{1-0}}{1-a^{3 p-3}} \cdot \frac{\gamma}{2^{3} a^{3 p}}\|x\|^{3 p},
$$




$$
\|g(x)-C(x)\| \leq \frac{a^{3 p-3}}{1-a^{3 p-3}} \frac{\gamma}{8 a^{3 p}}\|x\|^{3 p} \leq \frac{\|x\|^{3 p} \cdot \gamma}{8} \cdot \frac{1}{a^{3}-a^{3 p}} .
$$

Case (vi). $L=a^{3-3 p}, p>1, i=1$,

$$
\begin{aligned}
& \|g(x)-C(x)\| \leq \frac{L^{1-i}}{L} \beta(x) \leq \frac{\left(a^{3-3 p}\right)^{1-1}}{1-a^{3-3 p}} \frac{\gamma}{2^{3} a^{3 p}}\|x\|^{3 p} \\
& \|g(x)-C(x)\| \leq \frac{1}{1-a^{3-3 p}} \frac{\gamma}{8 a^{3 p}}\|x\|^{3 p} \leq \frac{\|x\|^{3 p} \cdot \gamma}{8 a^{3 p}} \cdot \frac{1}{a^{3 p}-a^{3}} .
\end{aligned}
$$

Hence, the proof is completed.

\section{REFERENCES}

[1] J. Aczel and J. Dhomberes, Functional Equations in Several Variables, Cambridge University Press, New York, New Rochelle, Melbourne, Sidney, 1989.

[2] T. Aoki, On the stability of linear transformation in Banach spaces, J. Math. Soc. Japan 2 (1950), 64-66.

[3] M. Arunkumar, S. Murthy and G. Ganapathy, Solution and Stability of n-dimensional quadratic functional equation, ICCMSC 2012, Computer and Information Science (CIS), Springer Verlag-Germany, 238, 2012, 384-394.

[4] M. Arunkumar, S. Murthy and V. Govindan, General solution and generalized Ulam-Hyers stability of a generalized $n$-type additive quadratic functional equation in Banach spaces and Banach algebra: using direct and fixed point method, International Journal of Advanced Mathematical Sciences 3(1) (2015), 25-64.

[5] M. Arunkumar, S. Murthy, V. Govindan and T. Namachivayam, General solution and four types of Ulam-Hyers stability of $n$-dimensional additive functional equation in Banach and fuzzy Banach spaces: Hyers direct and fixed point methods, International Journal of Applied Engineering and Research 11(1) (2016), 324-338.

[6] M. Arunkumar, K. Ravi and M. J. Rassias, Stability of a quartic and orthogonally quartic functional equation, Bull. Math. Anal. Appl. 3(3) (2011), 13-24.

[7] M. Arunkumar, S. Murthy and G. Ganapathy, Stability of a functional equation having nth order solution in generalized 2-normed spaces, International Journal of Mathematical Sciences and Engineering Applications 5(4) (2011), 361-369.

[8] T. Bag and S.K. Samanta, Finite dimensional fuzzy normed linear spaces, The Journal of Fuzzy Mathematics 11(3) (2003), 687-705.

[9] D. G. Bourgin, Classes of transformations and bordering transformations, Bull. Amer. Math. Soc. 57 (1951), 223-237.

[10] S. C. Chang and J. N. Mordeson, Fuzzy linear operator and fuzzy normed linear spaces, Bull. Calcutta Math. Soc. 86 (1994), 429-436.

[11] P. W. Cholewa, On the stability of quadratic functional mappings in normed spaces, Abh. Math. Semin. Univ. Hambg. 62 (1992), 59-64.

[12] S. Czerwik, Fundamental Equations and Inequalities in Several Variables, World Scientific, River Edge, NJ, 2002.

[13] C. Czerwik, On the stability of the quadratic mapping in normed spaces, Abh. Math. Semin. Univ. Hambg. 62 (1992), 59-64.

[14] P. Guvruta, A generalization of the Hyers-Ulam-Rassias stability of approximately additive mappings, J. Math. Anal. Appl. 184 (1994), 431-436.

[15] D. H. Hyers, On the stability of linear functional equations, Proc. Natl. Acad. Sci. USA 27 (1941), 222-224. 
[16] D. H. Hyers, G. Issac and Th.M. Rassias, Stability of Functional Equations in Several Variables, Birkhauser, Basel, 1998.

[17] K. W. Jun and H. M. Kim, The generalized Hyers-Ulam-Rassias stability of cubic functional equation, J. Math. Anal. Appl. 274(2) (2002), 267-278.

[18] S. M. Jung, Hyers-Ulam-Rassias Stability of Functional Equations, Mathematical Analysis, Hadronic Press, Palm Harbor, 2001.

[19] S. S. Jin and Y. H. Lee, Fuzzy stability of a general quadratic functional equation deriving from quadratic and additive mappings, Abstr. Appl. Anal. 2011 (2011), Article ID 534120, 15 pages.

[20] S. S. Jin and Y. H. Lee, Fuzzy stability of a general quadratic functional equation, Adv. Fuzzy Syst. 2011 (2011), Article ID 791695, 9 pages.

[21] S. S. Jin and Y. H. Lee, Fuzzy stability of the Cauchy additive and quadratic type functional equation, Commun. Korean Math. Soc. 27 (2012), 523-535.

[22] W. Liguang, L. Bo and B. Ran, Stability of a mixed type functional equation on multi-Banach spaces: a fixed point approach, J. Fixed Point Theory Appl. (2010), Article ID 283827, 9 pages.

[23] W. Liguang, The fixed point method for intuitionistic fuzzy stability of a quadratic functional equation, Fixed Point Theory Appl. (2010), Article ID 107182, 7 Pages.

[24] W. Liguang, and L. Bo, The Hyers-Ulam stability of a functional equation deriving from quadratic and cubic functions, in quasi- $\beta$-normed spaces, Acta Math. Sin. (Engl. Ser.) 26(12) (2010), 2335-2348.

[25] W. Liguang and L. Jing, On the stability of a functional equation deriving from additive and quadratic functions, Adv. Differential Equations 2012(98) (2012), 12 pages.

[26] W. Liguang, K. Xu and Q. Liu, On the stability a mixed functional equation deriving from additive, quadratic and cubic mappings, Acta Math. Sin. (Engl. Ser.) 30(6) (2014), 1033-1049.

[27] S. H. Lee, S. M. Im and I. S. Hwang, Quadratic functional equations, J. Math. Anal. Appl. 307 (2005), 387-394.

[28] S. S. Kim, Y. J. Cho and A. White, Linear operators on linear 2-normed spaces, Glas. Math. 27(42) (1992), 63-70.

[29] S. Murthy, M. Arunkumar and G. Ganapathy, Solution and stability of n-dimensional cubic functional equation in F-spaces: direct and fixed point methods, Proceedings of International Conference on Mathematical Sciences, Chennai, Tamil Nadu, July 17-19, 2014, 81-88.

[30] Z. Lewandowska, Linear operators on a generalized 2-normed spaces, Bull. Math. Soc. Sci. Math. Roumanie (N.S.) 42(4) (1999), 353-368.

[31] A. Najati, The generalized Hyers-Ulam stability of a cubic functional equation, Turkish J. Math. 31 (2007), 1-14.

[32] J. H. Park, S. B. Lee and W. G. Park, Stability results in non Archimedean L-fuzzy normed spaces for a cubic functional equation, J. Inequal. Appl. (2012), 193-201.

[33] J. M. Rassias, On approximately of approximately linear mappings of linear mappings, J. Funct. Anal. 46 (1982), 126-130.

[34] Th. M. Rassias, On the stability of the linear mapping in Banach spaces, Proc. Amer. Math. Soc. 72(2) (1978), 297-300.

[35] K. Ravi and M. Arunkumar, Hyers-Ulam-Rassias stability of a quartic functional equation, International Journal of Pure and Applied Mathematics 34(2) (2007), 247-260.

[36] K. Ravi and M. Arunkumar, On a General solution of a quartic functional equation, J. Comb. Inf. Syst. Sci. 33(1) (2008), 373-386.

[37] F. Skof, Local properties and approximations of operators, Seminario Matematico e Fisico di Milano 53 (1983), 113-129.

[38] S. M. Ulam, A Collection of Mathematical Problems, Interscience Publisher, New York, 1960.

[39] S. M. Ulam, Problem in Modern Mathematics, Sciences Editions, John Wiley and Sons Inc. New York, 1969. 
${ }^{1}$ Department of Mathematics,

Sri Vidya Mandir Arts and Science College,

Uthangarai - 636 902, TAmil NADU, India

Email address: govindoviya@gmail.com .

${ }^{2}$ Department of Mathematics,

Government Arts and Science College (For Men),

KRISHNAGIRI - 635 001, TAMIL NADU, INDIA

Email address: smurthy07@yahoo.co.in.

${ }^{3}$ Department of Mathematics,

Adhiyamaan College of Engineering,

Hosur - 635 109, TAmil NADU, India

Email address: saravanan040683@gmail.com. 